\title{
AIAA-99-2143 \\ SPACE TESTING OF ELECTROMAGNETICALLY SENSITIVE MATERIALS FOR BREAKTHROUGH PROPULSION PHYSICS
}

\author{
Alan C. Holt, Senior Member \\ NASA Johnson Space Center ${ }^{\star}$, Houston, TX \\ Eric W. Davis, Ph.D. \\ National Institute for Discovery Science, Las Vegas, NV \\ Hal Puthoff, Ph.D. \\ Institute for Advanced Studies, Austin, TX
}

\begin{abstract}
Recently it has been experimentally demonstrated that non-local (instantaneous) communication between two beams of light (spin direction) can randomly occur. The effect is described as a consequence of the physics of quantum mechanics. Other research has experimentally demonstrated that the zero point radiation/fields (ZPF), which pervade space-time, can effectively be shielded so as to force two parallel plates together (Casimir effect). The NASA Marshall Space Flight Center is investigating the possibility that a spinning superconductor can cause a reduction in the weight of nearby objects. Recent ultrahigh-intensity, peta/eta-watt tabletop lasers have achieved extreme electric and magnetic fields, pressure, temperature and space-time curvature that can only be found close to a black hole horizon. Based on these research and experimental efforts, some examples of space experiment and materials/technology testing approaches have been examined to determine the feasibility and potential benefits of using the International Space Station (ISS) to address breakthrough propulsion physics and technology challenges. The ISS's microgravity environment, combined with the access to an extreme vacuum and plasma environment, offers some unique advantages for the testing of various electromagnetically "sensitive" materials, and associated physical interactions. The use of this space laboratory could enable much more rapid progress in the identification and optimization of anomalous and highly nonlinear effects. A modular Breakthrough Propulsion Physics testbed could be developed using one or two mid-deck locker equivalent volumes in a pressurized Express Rack, which would enable the operation or testing of various experiments and devices as part of a long duration, breakthrough physics and technology testing program. Similarly, an Express Pallet adapter site could be used as a breakthrough propulsion physics testbed for those experiments or technology demonstrations which require direct access to the space environment and larger operating volumes. Breakthrough propulsion physics experiments could also be integrated into (or use extra space in or on) micro-/nano- technology (MNT) experiments which are already under development. The International Space Station's research capabilities provide important opportunities for achieving early breakthroughs in physics understanding, and the associated materials/technology interactions, needed to accelerate advances in space vehicle/platform maneuvering and transport.
\end{abstract}

\section{Introduction}

A number of recent advances in theoretical and experimental physics and materials development are providing insight into exploratory experiment approaches, which can be used to predict and look

* NASA (and its Breakthrough Propulsion Physics Program) supports the use of International Space Station's payload capabilities for breakthrough propulsion physics research described in this paper. NASA does not, however, endorse this paper."

Copyright (C) 1999 by the American Institute of Aeronautics and Astronautics, Inc. All rights reserved. for anomalies which could lead to substantial progress towards NASA's Breakthrough Propulsion Physics objectives. The International Space Station (ISS), which will soon begin to conduct a wide range of research activities, provides a unique environment for these type of exploratory investigations. In late 2000 and early 2001, the ISS will have the capability to install and operate pressurized payloads or experiments in the U.S. Laboratory.

The microgravity environment, access to an extremely low particle density (vacuum), and plasma characteristics of near-Earth orbit provide a unique laboratory testing capability. Using the pressurized Express Rack, mid-deck locker equivalent (MLE) volumes (8 MLEs per Express Rack), experiments can be quickly and easily installed, operated and returned 
(each Express Rack is about the size of a double-sized refrigerator). The use of the Express Rack capabilities would enable some unique exploratory testing of meso and micro-systems and materials in a microgravity environment, where detection and optimization of anomalous physical interactions could be more easily accomplished, in many cases.

Conducting early exploratory testing provides an opportunity to achieve an early breakthrough in propulsion physics, which would have a direct beneficial impact on technology development and testing for human exploration missions to Mars and elsewhere, and for probe missions being planned for the exploration of the outer planets and interstellar space. A recently released NASA Research Announcement may identify some space experiment proposals which could be candidates for early pressurized tests or later for externally attached or ISS-based free-flyer tests. ${ }^{1}$

This paper describes some examples of experiment testing approaches which could be, and perhaps should be, planned for in-space operations on the International Space Station. ${ }^{2}$ The examples are fairly generic, but they are based on specific ideas and experiment implementations which have been identified by or which are under consideration by the authors. We encourage other researchers to identify additional generic approaches and their own specific implementations to take advantage of future funding opportunities which may be provided by NASA, the Department of Defense and other organizations. Commercial offers by private companies could also encompass the testing and demonstration of breakthrough propulsion physics and technologies.

\section{Breakthrough Propulsion Research Paths}

\section{Zero-Point Energies and Fields}

Considerable progress has been made in the investigation of zero-point fields and energies over the past 10 years. The Casimir effect, which demonstrates real physical results of zero-point fields and energies, beyond the quantum effects, has been re-confirmed. Theoretical studies and models suggesting/proposing that zero-point fields (ZPF) may be primary causes of gravity and inertia have been very effectively described. ${ }^{3,4,5,6}$

Ground-based experiments to investigate the inertial effects of zero-point fields have been proposed and are in work. Approaches for extracting useful energy from zero-point fields have been proposed.,

The basic variable parameter behind the Casimir effect is the size of a cavity between two plates, which limit the wavelengths of zero-point fields or photons, which can exist between the plates. This limit results in a net difference in $\mathrm{ZPF}$ radiation pressure inside the cavity versus outside the cavity. The plates are forced together by this pressure difference.

Gravity, in the zero point field primary effects model, is caused by net pressure differences between points outside and inside a spherical mass, like the Earth for example. If this model is correct then it should be possible to create material/field configurations which, in effect, generate "cascading" cavities or conditions which either alter the net background pressure differences or which do not allow the background photons to interact with the mass of an object or vehicle. In other words, the effects of gravity are altered or manipulated.

Similarly, if inertia is caused by a zero-point field/photon "wind", then carefully designed experiments should be able to measure this effect, using rotating arms and sensitive detectors, for example. Tests of this kind are being conducted at the Institute for Advanced Studies in Austin, TX and perhaps elsewhere.

This theoretical model which links gravity, inertia and zero-point fields provides an opportunity to develop sensitive experiment configurations to test model predictions. Tests conducted in Earth orbit, microgravity conditions could result in anomaly identification and anomaly characterization, in a faster and more extensive manner than in comparable ground testing.

\section{Black Hole/Wormhole Physics}

Astrophysical studies are providing more and more significant evidence each year concerning the reality and effects of black holes. These stars, which have collapsed following supernovas into extremely dense and small volumes, with very high gravity fields (and in some cases magnetic fields), draw in the matter of nearby stars and warp space-time around them to the point that not even light can escape the gravity field. These effects are observable in spectral and imaging studies. The two salient features of black holes are their event horizons where gravity is so strong that captured light is forced into permanent non-escapable orbits and their singularities, where collapsed stellar matter and extreme gravitational tidal forces (or spacetime curvature) are located at an infinitesimal point.

In addition, theoretical studies in General Relativity have predicted the potential for man-made traversable wormholes to be formed by application of negative 
matter-energy fields. A traversable wormhole is essentially a tunnel through space-time connecting an entrance portal to an exit portal with the portals not possessing an event horizon and the tunnel not possessing a singularity. ${ }^{9}$ It is possible that man-made wormholes can be engineered to keep the gravitational tidal forces to a comfortable minimum.

Natural wormholes may be detected by astronomical searches for the gravitational lens effect they induce on background star light. ${ }^{10}$ If the conditions for wormholes exist in space, it may be possible to detect and eventually confirm their existence. In any case, we have some insight into techniques for artificially generating small wormholes which, according to present understanding, could in principle be used to send information faster than the speed of light, and perhaps eventually vehicles and beings as well. ${ }^{11,12,13}$

The distance to even the nearest star makes the prospects for human interstellar travel unappealing with current or even proposed advanced propulsion capabilities (except for those few individuals willing to spend most of their life on such a trip). In addition, getting the development and operations funding needed for a crewed interstellar vehicle requiring 30 to 50 years or more to complete its mission, would be extremely challenging, even with substantial commercial and international funding and interest. A breakthrough in propulsion, which as a minimum provides a propellentless propulsion augmentation, would help tremendously.

Interstellar probe missions venturing out into interstellar space can be accomplished without a major breakthrough in propellantless propulsion. However, a major physics breakthrough coupled with the quick development, testing and implementation of new propellantless propulsion technology could make a probe mission to another star system immediately feasible, and would jump-start a whole new round of Solar System probe exploration.

In recent testing, ultrahigh-intensity, peta/eta-watt tabletop lasers have achieved extreme electric and magnetic fields, pressure, temperature and space-time curvature that may only be found close to a black hole horizon. $^{14}$ In other experiments, non-local (instantaneous) communication between two beams of light (spin direction) has been experimentally demonstrated. The quantum mechanical coupling between the photons in the beam was random in these experiments. To be useful, the information must be able to be transmitted non-locally on a planned basis.
These experiments provide inspiration for the planning and conduct of experiments on the ground and perhaps in-space to take advantage of microgravity and other space environment conditions. The examples of space experiment approaches described below include one or more test approaches directed at expanding our understanding of the physics which may someday allow a vehicle and humans to be transported at speeds faster than the speed of light. Alcubierre has shown in his theoretical paper that by using the theory of General Relativity and negative matter-energy fields, it is possible to create a bubble around a vehicle, which would allow it to move at a velocity faster than the speed of light. In addition, no adverse differences in time or aging would be experienced by the participants, i.e. the travelers would not return to Earth to find no one they knew left alive. ${ }^{15}$

\section{Materials and Spinning and Pulsed Fields}

Great progress has been made in recent years in the development of new materials, at meso-, micro- and nano- scale levels. These materials demonstrate new properties and unusual reactions to various fields and forces. The discovery and production of carbon nanotubes $\left(\mathrm{C}_{60}\right.$ and other carbon configurations $)$ is one example of these new materials. Rice University and the NASA Johnson Space Center are jointly working together to study and improve the production of nanotubes for various space-related applications.

Our knowledge of extreme field/plasma interactions also continues to grow. Extensive studies and experimental research have been conducted in national and private laboratories (i.e., laser research, accelerator developments, fusion research, etc.); in-situ (i.e., upper atmosphere, magnetosphere and interplanetary space) and from ground and space observatories and instruments. The Earth's ionosphere/magnetosphere; the Sun's photosphere, chromosphere and corona; and distant astrophysical objects (stars, galaxies, and early universe epochs) have resulted in the identification of new phenomena, including massive energy sources which challenge our understanding of physics (gamma ray bursters, etc). The physics of the Sun's magnetic fields and their role in the generation of giant solar flare eruptions (magnetic field line merging and annihilation, etc.) continued to be studied and refined.

These research results are continuing to point to the importance of the configuration and motion of fields and plasmas in star systems, galaxies and the universe as a whole. Anomalies of field/plasma persistence have been observed in high-field-strength, plasma systems tested at national laboratories. The phenomena of ball 
lightning continues to be studied in an attempt to explain its formation and structure.

Spinning superconductors have been reported to cause a loss of gravity or weight in an object and are currently being experimentally studied at the NASA Marshall Space Flight Center (MSFC) in support of the NASA Advanced Space Transportation Research Program (lead by John Cole at NASA MSFC), and in support of the Breakthrough Propulsion Physics Program (lead by Marc Millis at the NASA Glenn Research Center). Researchers have also studied and reported anomalies in spinning and charged devices/materials in connection with other experiments involving superconductors and exotic materials.

These anomalies indicate that our understanding of physics is incomplete and still growing. By experimentally exploring precise, temporally and spatially varying configurations of fields, plasmas and materials, we might gain some unique insight into hidden nonlinearities of physical interactions. Extreme, but precise, changes and oscillations of fields, field strengths and energy flows could cause interactions or resonances currently unknown to us. This an area worth exploring in a systematic and carefully planned and instrumented manner. This type of exploratory approach or experimentation can result in much quicker progress in the detection and identification of anomalies which could lead to early breakthroughs in propellantless propulsion physics. At this stage, even negative results from well-planned experiments using sensitive instrumentation can be very beneficial.

\section{Breakthrough Propulsion Physics Testbeds (BPPT)}

\section{Express Rack Locker - BPPT-EXRACK}

There are two approaches, which can be effectively pursued for an Express Rack, Breakthrough Propulsion Physics testbed. One or two dedicated lockers can be configured for a capability to test a variety of approaches for identifying and optimizing anomalies that have breakthrough propulsion implications. Or, one or more experiments/devices can be integrated in with or use excess volume and resources of a payload or experiment already proposed for (or manifested on) an Express Rack.

Generic measurement technologies could include the use of laser systems with fringe interference measurement capabilities to accurately measure and detect anomalies. One or two techniques for measuring small changes in the gravitational field or the mass of a test mass/device should be provided. Sensitive electric and magnetic field sensors should be included to look for anomalies which are secondary effects of gravity altering or manipulation effects, including low to high frequency electromagnetic wave detectors. CCD cameras to record images with both white light and IR filters should also be included.

\section{Express Pallet-BPPT-EXPAL}

On flight UF3, currently scheduled for the year 2002, Express Pallets with a capability to support up to six separate payloads, will begin to be installed on the long exterior truss of the ISS. A generic breakthrough propulsion physics testbed could be developed to occupy one of the Express Pallet sites or to share a site with other technology test payloads. Measurement technologies could include some of those used in the Express Rack testbed and additional sensors, which characterize the surrounding plasma vacuum environment and induced field/plasma changes caused by test devices. High-energy radiation detectors may also be more important for larger and higher strength test devices.

\section{ISS-Based Free-Flyer}

The International Space Station currently does not provide an ISS-based free-flyer for technology development and testing. At a workshop held in 1995 a wide range of uses for an ISS free-flyer were discussed, including technology testbed uses. ${ }^{16}$ One or more commercial offers could eventually be submitted to provide this kind of capability for the Space Station. The use of an ISS based free-flyer is either required or highly desirable for performance testing of propellantless propulsion technology, and for systems reliability assessments. Measurement technologies would primarily be environmental, along with navigational sensors and gravity and particle detectors.

\section{Examples of BPP Test Approaches}

The following are examples of Breakthrough Propulsion Physics testbed approaches that appear to be promising. Specific experiment implementations based on these and other approaches could also be used in support of or as part of a complement of nano- and micro- technology (MNT) experiments and technology tests. MNT experiments are in the process of being selected by the Department of Defense for payloads funded and approved through DoD's Space Experiment Review Board. Other MNT experiments/ payloads may also be proposed. 


\section{EXAMPLE A - Pulsed, Micro-Engineered Material Systems}

Materials with properties that change under exposure to various types and frequencies of electric and magnetic fields are configured in certain spatial topologies, using micro-engineering and other techniques. These material topologies are then exposed to certain frequencies of electromagnetic fields while in stationary and spinning reference frames. Anomalies in electromagnetic and gravity field configurations and repeatable test device motions are then looked for. Once a motion or gravity anomaly is identified, parameters of the test bed and test device are varied to try to optimize the anomaly. Other field and spectral anomalies can also be clues to nonlinear instabilities or resonances, which could lead to useful motion or transport anomalies.

\section{EXAMPLE B - Zero Point Fields/Superconducting Energy State Suppression/Saturation Systems}

Using a combination of multiple layers of materials arranged in specific topologies, this test device attempts to leverage the zero-point field, cavity exclusion effects observed in the Casimir effect, to attempt to screen out or reduce the gravitational force or to artificially generate an accelerated reference frame in a specific direction. This approach can be augmented by the use of superconductors and other materials in which supersaturation of photonic energy levels is achieved, possibly enhancing unusual Casimir-like effects and hopefully resulting in gravity reduction or manipulation effects.

\section{EXAMPLE C - Sound-and-Light-Patterned Systems}

These test devices are established to test the geometric and other effects of sound and photon energy patterns resulting from their interaction with ionized gases (plasmas) and topological material mediums. Media with variable indices of refraction, sound patterns which modulate laser beams, and potential beat and resonance effects with zero point energy photons could be studied in this approach.

\section{EXAMPLE D - Plasma Cavities/Field Resonance Systems}

For these test devices the focus is on achieving spatial and temporal topologies of electromagnetic (EM) energy, EM energy gradients, and energy flow gradients which attempt to create resonances with background zero-point fields, higher order energy systems and field/energy relationships, which residually remain from the universe's early epoch transition from a single unified force at the Big Bang to the multiple forces we observe today. The resonances are essentially short-cuts to manipulating gravity and space-time structures.

\section{EXAMPLE E - Wormhole Characteristics/Extreme Field Strengths/Poynting Vector Systems}

Test devices are used to achieve extreme field intensities and gravitational anomalies at micro and nano scales to investigate gravity and space-time structure characteristics and relationships to electromagnetic fields and particles. Techniques for achieving similar effects in materials or dense plasma systems, which could lead to large scale collective gravity and space-time effects, would be investigated.

\section{Follow-On Technology Demonstration Scenarios}

Once anomalies have been identified and optimized through modeling and experimental work, a technology development and testing program can proceed. Again, space testing and demonstration of propellantless propulsion technology offers some distinct advantages because of the microgravity and plasma vacuum environment of space. For example, very low effective forces can be used to transport large masses in microgravity conditions - the one-g gravity at the Earth's surface is much harder to overcome, and the consequences of loss of power or transport force can cause considerable damage to a test vehicle. The risk in space for a technology payload can be much less if designed properly (i.e., to avoid any runaway condition).

For the benefit of discussing possibilities for follow-on research, let's assume that a propulsion physics breakthrough has been achieved. The breakthrough would lead to the development of a computer based, mathematical physics model which could be used to develop, integrate and test advanced system propulsion, guidance, navigation and control technologies.

This propellantless propulsion technology capability could be tested first at an external, attached site to insure compatibility with space environmental effects, to test sub-system reliability and to better categorize guidance, navigation and control $(\mathrm{GN} \& \mathrm{C})$ parameters. Low-magnitude force vectors generated during these tests could be directed through the ISS's center of mass, and operated during a time when ISS can gain from the additional force (for re-boost, atmospheric drag cancellation, etc.). 
The next step would be to mount a propellantless propulsion system on a free-flyer which is either based at the station or which frequently visits the station. The propellantless propulsion system would be activated and tested only at safe distances from the station. Low to medium level force vector tests could be accomplished on ISS-based, local free-flyers, but to expand the performance envelope, larger free-flyers (possibly commercially provided) would be needed. In both cases, the technology could be retrieved from the platform by ISS robotics and returned to the ground, for analysis and refurbishment/refinements.

While a human flight to Mars can be accomplished without a propulsion breakthrough, any breakthrough which can be cost-effectively implemented could, as a minimum, provide a high performance back-up to a primary Mars transport propulsion system. Therefore, an additional testing opportunity could be provided on a Mars transport prototype or test vehicle assembled and tested at or near the International Space Station, or later on an operational transport vehicle. Once a propellantless propulsion's system reliability and performance is demonstrated and crew safety qualified, it then can become a primary candidates for any upgrades planned for Mars transport vehicles and for new, deep space human mission and interstellar probe mission vehicles.

We would expect to be successful in finding and developing ways to manipulate gravity/space-time for sub-light, propellantless propulsion much sooner than we would for faster-than-light travel. Nevertheless, a breakthrough which could ultimately enable practical faster-than-light transport could occur at any time. Since anything other than micro- or nano- size space warps could have challenging safety concerns, the use of an ISS-visiting (using conventional propulsion for rendezvous and capture), but deep-space free-flyer would likely be required for faster-than-light performance testing.

Propellantless propulsion capabilities for sub-light and faster-than-light transport could also be tested on a small free-flyer which is deployed from a larger free-flyer (for example, something the size of the ball- shaped AERCAM which was tested in the cargo bay of the Space Shuttle). Small deployables could also be released from a deep space transport testbed (i.e., Mars transport prototype) or from a Mars transport vehicle actually on the way to or from Mars.

While the testing of propellantless propulsion systems at or near the ISS would by design not cause any detrimental effects to the crew and other ISS systems, full-up performance testing of propellantless propulsion systems which manipulate gravity and/or space-time would need to include in-depth assessment of effects on the crew. Access to or proximity to the International Space Station, where access exists to extensive human life research equipment, health maintenance facilities, and extensive logistics transport capability to and from the Earth, would be very desirable if not required for early testing phases.

\section{Summary}

The above examples of Breakthrough Propulsion Physics (BPP) testbed approaches represent areas of exploratory research which may be especially promising for space-experiment, propellantless propulsion research. Experiments and demonstrations on the ISS should enable NASA and the research community to make faster progress towards the identification and optimization of anomalies, which could lead to the development of large scale zero-point field energy and gravity/inertia effects, non-local communication and energy transfer effects (in a controlled manner) and other gravity/space-time manipulation effects.

Technology readiness assessments for human exploration, and planning for interstellar probe missions, demonstrate the importance and very high value which early exploratory attempts at breakthroughs in propellantless propulsion have, despite the low chances for success. The International Space Station, and its extensive research and technology testing capability, could very well be the catalyst for breakthroughs leading to technology developments which will enable humanity's first "star trek".

\section{References}

1. NASA Research Announcement for Breakthrough Propulsion Physics, SOL NRA-99-LERC-1.

2. Holt, A.(1998), "International Space Station technology demonstrations", in STAIF-98, 15 Symposium on Space Nuclear Power \& Propulsion AIP Conference Proceedings 420, ed. M.S. El-Genk.

3. Casimir, H. B. G. (1948), "On the Attraction Between Two Perfectly Conducting Plates", Proc. Kon. Ned. Akad. Wetenschap, 51, p. 793.

4. Puthoff, H. E. (1989), "Gravity as a zero-point fluctuation force", Phys. Rev. A, 39, p. 2333; Phys. Rev. A, 47, p.3454.

5. Haisch, B., Rueda, A. and Puthoff, H. E. (1994), "Inertia as a zero-point field Lorentz Force", Phys. Rev. A, 49, p. 678. 
6. Rueda, A. and Haisch, B. (1998), "Inertia as reaction of the vacuum to accelerated motion", Phys. Lett. A 240, 115.

7. Forward, R. L. (1984), "Extracting electrical energy from the vacuum by cohesion of charged foliated conductors", Physical Review, B30, p. 1770.

8. Puthoff, H.E. (1998), "Can the vacuum be engineered for spaceflight applications? Overview of theory and experiments," Jour. Sci. Exploration, 12, p.295.

9. Morris, M. and Thorne, K. (1988), "Wormholes in spacetime and their use for interstellar travel: A tool for teaching general relativity", Amer. J. Phys., 56, no. 5, pp. 395-412.

10. Cramer, J., Forward, R. L., Morris, M., Visser, M., Benford, G. and Landis, G. (1995), "Natural Wormholes as Gravitational Lenses", Physical Review D, pp. 3124-3127.

11. Davis, E. W. (1999), "Wormhole Induction Propulsion (WHIP)", in NASA Breakthrough Propulsion Physics Workshop Proceedings, NASA CP-1999-208694, pp. 157-163.
12. Davis, E. W. (1998), "Interstellar Travel By Means of Wormhole Induction Propulsion (WHIP)", in STAIF-98, $15^{\text {th }}$ Symposium on Space Nuclear Power and Propulsion, AIP Conference Proceedings 420, ed. M. S. El-Genk, pp. 1502-1508.

13. Van Flandern, T.(1998), "The speed of gravity What the experiments say", Phys. Lett A 250, 1-11.

14. Mourou, G. A., Barty, C. P. J. and Perry, M. D. (1998), "Ultrahigh-Intensity Lasers: Physics Of The Extreme On A Tabletop", Physics Today, 51, no. 1, pp. 22-28. See also the references cited therein.

15. Alcubierre, M. (1994), "The warp drive: hyper-fast travel within general relativity", Class. Quant. Grav., 11, L73.

16. International Space Station Free-Flyer Workshop, NASA International Space Station Office, Johnson Space Center, and the Goddard Space Flight Center, University of Maryland, College Park, Maryland, May 14-15, 1996. 